\title{
Elimination of apoptotic boar spermatozoa using magnetic activated cell sorting
}

\author{
Janko Mrkun ${ }^{1}$, Tamara Dolenšek², Tanja Knific², Anja Pišlar ${ }^{3}$, Marjan Kosec $^{1}$, \\ Janko Kos ${ }^{3,4}$, Petra Zrimšek ${ }^{1}$ \\ ${ }^{1}$ University of Ljubljana, Veterinary Faculty, Clinic for Reproduction and Horses, Slovenia \\ ${ }^{2}$ Students of the final year at Veterinary Faculty, ${ }^{3}$ Faculty of Pharmacy, Ljubljana, Slovenia \\ ${ }^{4}$ Institute Jožef Stefan, Department of Biotechnology, Ljubljana, Slovenia
}

Received February 21, 2013

Accepted December 4, 2013

\begin{abstract}
One of the features of apoptosis is the externalization of phosphatidylserine which could be used to remove apoptotic cells from semen preparations. Magnetic-activated cell sorting using annexin V-conjugated microbeads which bind to phosphatidylserine could be used to enhance semen quality. Twelve boar semen samples after 3 days of liquid storage at $16-17^{\circ} \mathrm{C}$ were subjected to magnetic-activated cell sorting. Bound and unbound fractions and control samples were subjected to flow cytometry following the staining of spermatozoa with Annexin V conjugated with Alexa Fluor 488 and propidium iodide. Four subpopulations were obtained: live, early apoptotic live, late apoptotic, early necrotic dead and late necrotic dead. The frequency of early apoptotic and late necrotic spermatozoa was significantly higher $(P<0.05)$ in bound $(14.1 \pm 10.6 \%$ and 24.1 $\pm 10.2 \%$, respectively) than in unbound fractions $(3.4 \pm 2.1 \%$ and $12.7 \pm 3.1 \%)$ and control $(3.5 \pm 1.6 \%$ and $12.0 \pm 5.0 \%)$. The lowest concentration of live spermatozoa was found in the bound fraction $(10.6 \pm 8.0 \%)$, which differed significantly $(P<0.05)$ from the control. In unbound fractions there was a significantly higher concentration $(P<0.05)$ of morphologically normal spermatozoa $(31.8 \pm 12.6 \%)$ compared to bound ones $(5.9 \pm 7.3 \%)$. A significantly $(P<0.05)$ lower proportion of morphologically normal spermatozoa was observed in both fractions compared to control $(67.2 \pm 17.0 \%)$. Boar spermatozoa were separated by the above method for the first time, however, the results showed this method to be inappropriate for boar semen separation under the tested conditions.
\end{abstract}

Semen, short-term storage, MACS, apoptosis

The fertility of boar spermatozoa decreases after cryopreservation and with increased storage time during liquid preservation. During storage, the boar spermatozoa undergo several changes including diminished motility, viability, presence of matrix metalloproteases (Zakošek Pipan et al. 2010) and alterations in membrane permeability, together with apoptotic-like changes (Kumaresan et al. 2009). Apoptosis contributes to decreased fertility due to disruption of membrane, phospholipid asymmetry and externalization of the phospholipid phosphatidylserine (PS) that is normally present on the inner leaflet of the plasma membrane (Vermes et al. 1995). Although capacitation and the acrosome reaction affect membrane properties such as ion permeability, surface charge, lectin binding, and protein phosphorylation (Flesh and Gadella 2000), PS does not become exposed on the outer surface of the viable boar spermatozoa (Kurz et al. 2005). Therefore, externalization of PS could be used to remove apoptotic-like spermatozoa from sperm preparations to enhance sperm quality in assisted reproduction technologies. Phospholipid phosphatidylserine has a high and selective affinity for the phospholipid-binding protein annexin V (van Heerde and de Groot 1995), therefore magnetic-activated cell sorting (MACS) using annexin V-conjugated superparamagnetic microbeads was established to separate non-apoptotic human spermatozoa from apoptotic ones (Grunewald et al. 2001). In veterinary medicine, MACS has been used for eliminating apoptotic spermatozoa from rabbit insemination dose (Vasicek et al. 2011) and studied as a possible technique for preparing enriched populations of bovine spermatogonia (Herrid et al. 2009), but no such studies included boar spermatozoa.

Address for correspondence:

Assoc. Prof. Petra Zrimšek

Clinic for Reproduction and Horses

Veterinary Faculty, University of Ljubljana

Gerbičeva 60, Ljubljana, Slovenia
Phone: +38614779271
Fax: +386 12832243
E-mail: petra.zrimsek@vf.uni-lj.si
http://actavet.vfu.cz/ 
The aim of our study was to evaluate the application of MACS, using annexin V-conjugated microbeads for separating apoptotic spermatozoa from boar semen.

\section{Materials and Methods}

\section{Experimental design}

Twelve semen samples collected during a routine examination from 12- to 24-month-old boars of various breeds (Slovenian Landrace, Slovenian Large White, and Pietrain) with proven fertilizing ability were used in this study. While the boar mounted a dummy sow, semen was collected with a gloved hand using a clean semen collecting flask that filters gel, dust and bristles out. Semen was then immediately diluted (1:2) with Beltswille Thawing Solution (Truadeco, Netherlands) and kept at room temperature during transportation to the laboratory. The samples were stored for 3 days at $16-17{ }^{\circ} \mathrm{C}$ with constant, gentle agitation. The semen suspension of each sample was divided into two aliquots, one serving as a control and the other subjected to magnetic cell sorting (MACS) using the Annexin V Microbead Kit (Miltenyi Biotec, GmbH, Bergisch Gladbach, Germany). The analysis of sperm was focused on their concentration, motility and morphology characteristics and comparison of Annexin V/propidium iodide (PI) staining of control semen samples and sperm fractions after MACS separation.

\section{Isolation of spermatozoa by magnetic-activated cell sorting}

Semen samples containing $5 \times 10^{7}$ spermatozoa were centrifuged at $500 \mathrm{~g}$ for $10 \mathrm{~min}$ at room temperature, the supernatant was removed and the sperm pellet resuspended in $\times 1$ binding buffer (Annexin V Microbead Kit, Germany). Solution containing annexin V-conjugated microbeads (Annexin V Microbead Kit) was added at the amount of $100 \mu \mathrm{l}$ and the suspension was incubated for $15 \mathrm{~min}$ at room temperature with constant gentle agitation to perform magnetic labelling. A MS column containing iron balls was first rinsed with binding buffer and fitted in a miniMACS separator (magnetic field) attached to a multistand. The spermatozoa-microbead suspension was added to the top of the column and the unbound fraction containing spermatozoa with intact membranes eluted from the column. The column was then removed from the magnetic field and the suspension eluted with binding buffer. The bound fraction contained spermatozoa with externalized PS which bound to annexin V microbeads. Bound and unbound fractions were centrifuged for $10 \mathrm{~min}$ at $500 \mathrm{~g}$ at room temperature. The supernatant was removed and the semen pellet was resuspended in Beltsville Thawing Solution to provide optimal conditions for boar spermatozoa.

Sperm concentration, motility, and morphology

Computer assisted semen analysis (CASA; Hamilton Thorne IVOS 12.3; Hamilton Thorne Research, MA, USA) was performed with a Makler counting chamber (Sefi Medical Instruments, Israel) to determine sperm concentration and motility characteristics. The parameters were set as follows: minimum area of spermatozoa's head -7 pixels, velocity average path (VAP) $-20 \mu \mathrm{m} / \mathrm{s}$ and velocity straight line (VSL) $-5 \mu \mathrm{m} / \mathrm{s}$.

Thin smears of the well-mixed semen were prepared in duplicate for determining semen morphology. The slides were air-dried, then stained with Giemsa dye (Merck, Germany). A total of 200 spermatozoa were scored per slide, except on slides from bound fractions where 100 spermatozoa or less were scored due to the low concentration of spermatozoa in these fractions. Bright field illumination and an oil immersion objective with a total magnification of $\times 1000$ were used. The spermatozoa were categorized in the following groups: normal, detached acrosome, acrosome in detachment, deformed acrosome, abnormal head, cytoplasmic droplet, midpiece defect, coiled tail and tail defect.

\section{Flow cytometry analysis}

For determining spermatozoa with flow cytometry, Vybrant Apoptosis Assay Kit \#2 (Invitrogen, Great Britain) was used. Sperm samples were diluted $\times 5$ in binding buffer. An aliquot of $100 \mu \mathrm{l}$ from the prepared solution was stained with $5 \mu \mathrm{l}$ Alexa Fluor 488 - Annexin V solution and $1 \mu \mathrm{l}$ PI solution and then incubated for $15 \mathrm{~min}$ at room temperature. Binding buffer $(300 \mu \mathrm{l})$ was added to control samples and samples from the unbound fraction, and $150 \mu \mathrm{l}$ of binding buffer to the samples of bound fractions, due to the low sperm concentration in these fractions. Samples were then placed on ice and analyzed within $1 \mathrm{~h}$ using FACS Calibur flow cytometer (BD Biosciences, San Jose, CA, USA). From each spermatozoa forward light scatter (FSC), orthogonal light scatter (SSC), Annexin V - Alexa Fluor 488 fluorescence (FL1), and PI fluorescence (FL3) was evaluated using Flow Jo software (Ashland, OR, USA). A gate was applied in the FCS/SSC dot-plot to restrict the analysis of spermatozoa. For the gated cells, the percentages of Annexin- $\mathrm{V}$ negative and positive (A- and A+), PI negative and positive (PIand $\mathrm{PI}+$ ), double positive and double negative were evaluated, based on quadrants determined from single-stained and unstained control samples. Four sperm subpopulations were revealed: R1: A-/PI- (live), R2: A+/PI- (early apoptotic, live), R3: A+/PI+ (late apoptotic and early necrotic, dead), R4: A-/PI+ (late necrotic, dead).

\section{Statistical analysis}

Differences between semen parameters of control samples and unbound and bound fractions were compared using One Way Analysis of Variance in the case of normal distribution, and equality of the variances and Kruskal-Wallis Analysis of Variance on Ranks in case of non-normal distribution and non-equal variances. When 
a significant difference was obtained, values were compared by parametric Tukey test or non-parametric HolmSidak method. SigmaStat 3.5 (SYSTAT Software Inc.) software was used for the above analysis. $P<0.05$ was considered as significant.

\section{Results}

\section{Flow cytometry with Annexin V and propidium iodide staining}

The mean sperm concentration in control samples was $119.8 \pm 46.9 \times 10^{6} / \mathrm{ml}$. Spermatozoa were subjected to MACS separation, resulting in a mean of $83.2 \pm 27.6 \%$ spermatozoa in unbound fractions.

Fig. 1 (Plate II) shows two dimensional plots of fluorescence intensities of Annexin V-Alexa Fluor and PI of control (C), unbound (A1) and bound fractions (A2) obtained by flow cytometric assessment. The percentage of gated cells did not differ between samples; it was $83.5 \pm 4.7 \%, 79.0 \pm 9.4 \%$ and $83.2 \pm 7.6 \%$ in control, unbound and bound fractions, respectively (Table 1). The frequency of early apoptotic (A+/PI-) and late necrotic (A-/ $\mathrm{PI}+)$ spermatozoa was significantly $(P<0.05)$ higher in bound fractions than in unbound fractions and control. The lowest concentration of live spermatozoa was found in bound fractions where it differed significantly $(P<0.05)$ from control. No significant differences $(P>0.05)$ were observed between unbound fractions and control (Table 1).

Table 1. Subpopulations of boar sperm in samples obtained by flow cytometric assessment.

\begin{tabular}{lccc}
\hline Subpopulations & Control & Unbound fraction & Bound fraction \\
\hline R1 (\%) & $32.9 \pm 20.2^{\mathrm{a}}$ & $18.8 \pm 12.2^{\mathrm{ac}}$ & $10.6 \pm 8.0^{\mathrm{bc}}$ \\
R2 (\%) & $3.5 \pm 1.6^{\mathrm{a}}$ & $3.4 \pm 2.1^{\mathrm{a}}$ & $14.1 \pm 10.6^{\mathrm{b}}$ \\
R3 (\%) & $37.6 \pm 17.7^{\mathrm{a}}$ & $48.6 \pm 16.0^{\mathrm{ab}}$ & $30.2 \pm 15.0^{\mathrm{ac}}$ \\
R4 (\%) & $12.0 \pm 5.0^{\mathrm{a}}$ & $12.7 \pm 3.1^{\mathrm{a}}$ & $24.1 \pm 10.2^{\mathrm{b}}$ \\
Gated cells (\%) & $83.5 \pm 4.7^{\mathrm{a}}$ & $79.0 \pm 9.4^{\mathrm{a}}$ & $83.2 \pm 7.6^{\mathrm{a}}$ \\
\hline
\end{tabular}

Sperm subpopulations: R1 - A-/PI- (live), R2 - A+/PI- (early apoptotic, live), R3 - A+/PI+ (late apoptotic and early necrotic, dead), R4 - A-/PI+ (late necrotic, dead). Data are expressed as mean \pm SD, a,b,c - values marked with different superscripts mean that there are significant differences $(P<0.05)$ in rows.

Table 2. Motility and morphological characteristics of boar sperms after magnetic-activated cell sorting using annexin V-conjugated microbeads.

\begin{tabular}{lccc}
\hline & Control & Unbound fraction & Bound fraction \\
\hline Motility characteristics & $68.4 \pm 11.5^{\mathrm{a}}$ & $5.9 \pm 4.0^{\mathrm{b}}$ & NA \\
Motility (\%) & $26.6 \pm 15.9^{\mathrm{a}}$ & $0.9 \pm 0.8^{\mathrm{b}}$ & NA \\
Progressive motility (\%) & & & \\
Morphological characteristic* & $67.2 \pm 17.0^{\mathrm{a}}$ & $31.8 \pm 12.6^{\mathrm{b}}$ & $5.9 \pm 7.3^{\mathrm{c}}$ \\
Normal spermatozoa (\%) & $5.7 \pm 4.8^{\mathrm{a}}$ & $12.7 \pm 7.2^{\mathrm{a}}$ & $57.8 \pm 23.0^{\mathrm{b}}$ \\
Detached acrosome (\%) & $6.7 \pm .1^{\mathrm{a}}$ & $2.6 \pm 4.8^{\mathrm{b}}$ & $0.5 \pm 0.9^{\mathrm{b}}$ \\
Acrosome in detachment (\%) & $3.5 \pm 3.3^{\mathrm{a}}$ & $0.5 \pm 0.7^{\mathrm{b}}$ & $0.2 \pm 0.7^{\mathrm{b}}$ \\
Cytomplasmic droplet (\%) &
\end{tabular}

NA - not applicable (only individual sperms showed motility, results of bound fractions obtained by computer assisted semen analysis are irrelevant due to small number of spermatozoa in samples), *Only morphological characteristics that differ significantly between semen samples are listed. Data are expressed as mean \pm SD. a,b,c - values marked by the different superscripts in rows are significantly different $(P<0.05)$. 
Motility characteristics

Sperm motility and progressive motility decreased after MACS separation, showing significantly lower values in unbound fractions than in control (Table 2). In bound fractions, only individual sperm were viable and showed motility, but the results of bound fractions obtained by CASA are irrelevant due to the small number of spermatozoa in samples.

\section{Morphological abnormalities}

There was no significant difference $(P>0.05)$ between semen samples in terms of deformed acrosome, coiled tail, abnormal head, tail or midpiece. Morphological abnormalities that differed significantly between semen samples are listed in Table 2 . The proportion of normal spermatozoa observed in both fractions were significantly $(P$ $<0.05)$ lower than in control. Bound fractions contained significantly lower proportions of normal spermatozoa and significantly higher proportions of detached acrosomes than unbound ones $(P<0.05)$, although both fractions differed from controls. Acrosome detachment and cytoplasmic droplets were observed less frequently in bound and unbound fractions compared to control $(P<0.05)$. Bound and unbound fractions differed in terms of the abnormalities noted above, from controls $(P<0.05)$ but not from each other $(P>0.05)$.

\section{Discussion}

Magnetic-activated cell sorting, using annexin V-conjugated microbeads, enables nonapoptotic spermatozoa to be separated from apoptotic ones. However, in contrast to the successful separation of apoptotic human spermatozoa (Said et al. 2006; Polak de Fried and Denaday 2010), MACS caused damage to boar semen.

The MACS has been used successfully to enhance human semen quality for assisted reproductive techniques (Polak De Fried and Denaday 2010; Raw e etal.2010). Selecting non-apoptotic human spermatozoa in assisted reproduction using intracytoplasmic sperm injection (ICSI) improved the outcome in terms of fertilization, cleavage, pregnancy and implantation rates (Dirican et al. 2008). It also enhances the percentage of spermatozoa with intact transmembrane mitochondrial potential and mitochondrial integrity survival rates following human semen cryopreservation (Grunewald et al. 2006). In veterinary medicine only rabbit spermatozoa have so far been separated by MACS (Vasicek et al. 2011). In the present study, boar spermatozoa have been separated by MACS for the first time. Flow cytometry, following staining with Annexin V-conjugated with Alexa Fluor 488 and propidium iodide, enabled complex analysis of four subpopulations according to membrane integrity. Although early apoptotic and late necrotic (dead) spermatozoa were found in all semen samples, their frequency was significantly higher in bound than in unbound fractions and control, confirming the successful binding of microbeads to apoptotic spermatozoa. In human semen following MACS, all subpopulations were present in bound as well as unbound fractions, but differences were significantly greater (Lee et al. 2010), as in our study.

A significantly higher level of morphologically normal spermatozoa and a lower percentage of detached acrosome were observed in unbound fractions compared to bound ones, in agreement with the results of MACS separation in human semen (Aziz et al. 2007). In both bound and unbound fractions, lower levels of acrosome detachment are observed, due to the higher proportion of spermatozoa with detached acrosome. The percentage of detached acrosome increased after MACS, especially in bound fractions, whereas the percentage of acrosome in detachment decreases in all fractions compared to control samples. After separation, the level of morphological abnormalities was significantly higher in all fractions compared to control samples, indicating that morphological changes 
of spermatozoa occurred during MACS. This finding contrasts with the results obtained for human semen, where a higher level of morphologically normal spermatozoa is observed in unbound fractions compared to control samples after MACS separation (Grunewald et al. 2006; Said et al. 2006; Aziz et al. 2007). In other studies, however, human spermatozoa were not evaluated according to their morphology (De Vantery Arrighi et al. 2009; Lee et al. 2010) and no information on morphological changes is available for rabbit spermatozoa following MACS (Vasicek et al. 2011). The majority of authors report an increase of human sperm motility in unbound fractions after MACS separation (De Vantery Arrigi et al. 2009; Rawe et al. 2010). Although sperm motility is slightly decreased after MACS, most sperm characteristics in human semen remain impaired (Le e et al. 2010). In unbound fractions of boar semen, motility decreased significantly compared to control samples, whereas in bound fractions only several spermatozoa were motile. Centrifugation regime and temperature used for incubation of spermatozoa with MicroBeads were adapted for boar sperm, therefore, a large decrease in motility is proposed due to the application of the MACS procedure to boar semen. The reason could be that the boar sperm membrane is more susceptible to the MACS procedure than that of human spermatozoa. Also, exposure to the magnetic field was found as a biologically toxic influence to spermatogenesis (Saadeldin et al. 2011).

In conclusion, the use of MACS for separation of apoptotic and dead cells caused damage to the boar spermatozoa in terms of motility, viability and morphology. Therefore, MACS appears to be inappropriate for the separation of boar semen under the tested conditions, although it has been used successfully for excluding apoptotic spermatozoa from human semen.

\section{Acknowledgements}

This work was supported by the Slovenian Research Agency, "Endocrine, immune, nervous and enzyme responses in healthy and sick animals" (P4-0053). Authors thank Mateja Bogataj for technical assistance in semen analysis using CASA and Prof. Roger Pain for the English revision of the manuscript.

\section{References}

Aziz N, Said T, Paasch U, Agarwal A 2007: The relationship between human sperm apoptosis, morphology and sperm deformity index. Hum Reprod 22: 1412-1419

De Vantery Arrighi C, Lucas H, Chardonnens D, de Agostini A 2009: Removal of spermatozoa with externalized phosphatidilyserine from sperm preparation in human assisted medical procreation: effects on viability, motility and mitochondrial membrane potential. Reprod Biol Endocrinol 7: 1

Dirican EK, Ozgün OD, Akarsu S, Akin KO, Ercan O, Uğurlu M, Camsari C, Kanyilmaz O, Kaya A, Unsal A 2008: Clinical outcome of magnetic activated cell sorting of non-apoptotic spermatozoa before density gradient centrifugation for assisted reproduction. J Assist Reprod Genet 25: 375-381

Flesch FM, Gadellam BM 2000: Dynamics of the mammalian sperm plasma membrane in the process of fertilization. Biochim Biophys Acta 1469: 197-235

Grunewald S, Baumann T, Paasch U, Glander HJ 2006: Capacitation and acrosome reaction in nonapoptotic human spermatozoa. Ann NY Acad Sci 1090: 138-146

Grunewald S, Paasch U, Glander HJ 2001: Enrichment of non-apoptotic human spermatozoa after cryopreservation by immunomagnetic cell sorting. Cell Tissue Bank 2: 127-133

Herrid M, Davey RJ, Hutton K, Colditz IG, Hill JR 2009: A comparison of methods for preparing enriched populations of bovine spermatogonia. Reprod Fertil Dev 21: 393-399

Kumaresan A, Kadirvel G, Bujarbaruah KM, Bardoloi RK, Das A, Kumar S, Naskar S 2009: Preservation of boar semen at $18^{\circ} \mathrm{C}$ induces lipid peroxidation and apoptosis like changes in spermatozoa. Anim Reprod Sci 110: $162-171$

Kurz A, Viertel D, Herrmann A, Müller K 2005: Localization of phospatidylserine in boar sperm cell membranes during capacitation and acrosome reaction. Reproduction 130: 615-626

Lee TH, Liu CH, Shih YT, Tsao HM, Huang CC, Chen HH, Lee MS 2010: Magnetic-activated cell sorting for sperm preparations reduces spermatozoa with apoptotic markers and improves the acrosome reaction in couples with unexplained infertility. Hum Reprod 25: 839-846

Polak de Fried E, Denaday F 2010: Single and twin ongoing pregnancies in two cases of previous ART failure after ICSI performed with sperm sorted using annexin V microbeads. Fertil Steril 351: e15-351e18 
Rawe VY, Boudri HU, Alvarez Sedo C, Carro M, Pappier S, Nodar F 2010: Healthy baby born after reduction of sperm DNA fragmentation using cell sorting before ICSI. Reprod Biomed Online 20: 320-323

Said T, Agarwal A, Grunewald S, Rasch M, Baumann T, Kriegel C, Li L, Glander HJ, Thomas AJ Jr, Paasch U 2006: Selection of nonapoptotic spermatozoa as a new tool for enhancing assisted reproduction outcomes: an in vitro model. Biol Reprod 74: 530-537

Saadeldin IM, Fadel AM, Hamada MMZ, El-Badry AA 2011: Effects of exposure to $50 \mathrm{~Hz}, 1$ Gauss magnetic field on reproductive traits in male albino rats. Acta Vet Brno 2011 80: 107-111

Vasicek J, Parkanyi V, Ondruska L, Makarevich A, Chrenek P 2011: Elimination of apoptotic spermatozoa from rabbit insemination dose using annexin V associated with the MACS technique. Folia Biol 59: 65-69

Van Heerde WL, de Groot PG, Reutelingsperger CP 1995: The complexity of the phospholipid binding protein annexin V. Thromb Haemost 73: 172-179

Vermes I, Haanen C, Steffens-Nakken H, Reutelingsperger CP 1995: A novel assay for apoptosis: flow cytometric detection of phosphatidylserine expression of early apoptotic cells using fluorescein labelled annexin V. J Immunol Methods 184: 30-151

Zakošek Pipan M, Kosec M, Mrkun J, Zrimšek P 2010: Gelatinases in boar seminal plasma and their relation to semen indicators. Acta Vet Brno 79: 491-496 
Plate II

Mrkun J. et al.: Elimination ... pp. 13-18

C

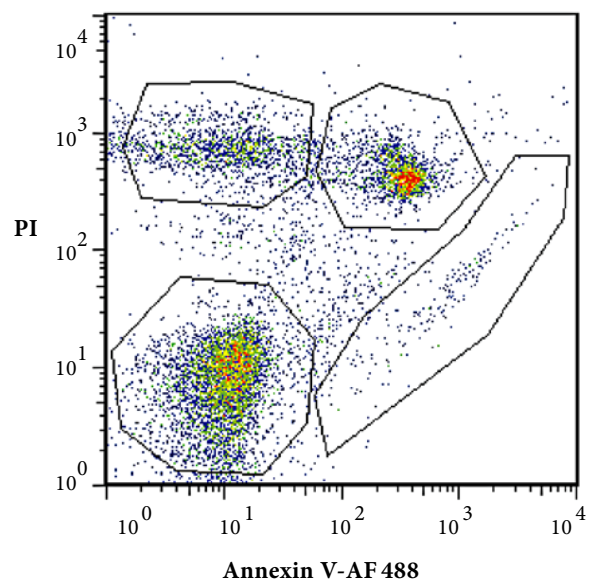

A1

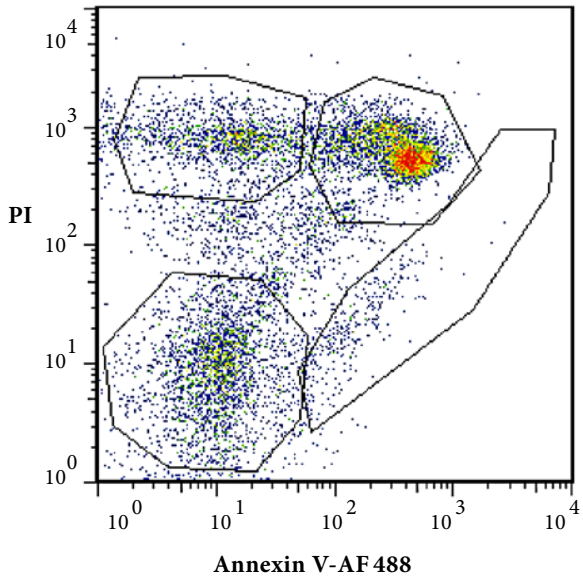

A2

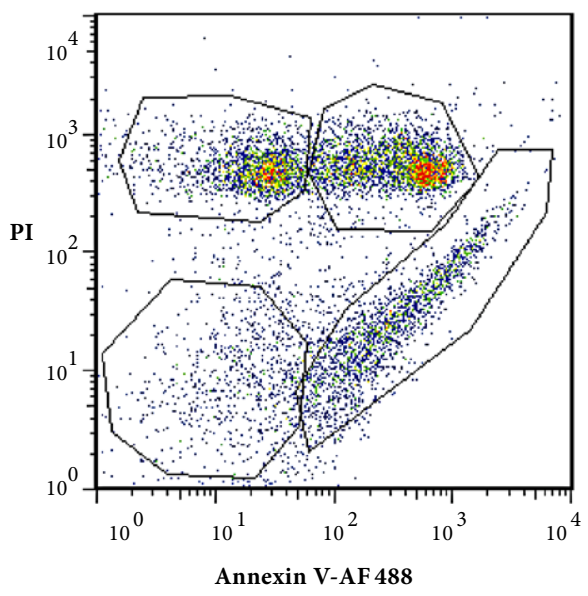

Fig. 1. Flow cytometric assessment of unbound and bound fractions of boar semen after magnetic-activated cell sorting using annexin V-conjugated microbeads

C - control sample, A1 - unbound fraction after magnetic-activated cell sortin using annexin V-conjugated microbeads, A2 - bound fraction after MACS using annexin V-conjugated microbeads, PI - detection of spermatozoa with bound propidium iodide (fluorescence detector FL3), Annexin V-AF488 - detection of spermatozoa bound to Annexin V-conjugated with Alexa Fluor 488 (fluorescence detector FL1), sperm subpopulations: R1 - A-/PI- (live), R2 - A+/PI- (early apoptotic, live), R3 - A+/PI+ (late apoptotic and early necrotic, dead), R4 - A-/PI+ (late necrotic, dead) 